La Revue

des Droits

de l'Homme

\section{La Revue des droits de l'homme}

Revue du Centre de recherches et d'études sur les droits fondamentaux

Actualités Droits-Libertés | 2020

\title{
Arrêt des traitements médicaux pour un patient âgé
}

Quelques réflexions à propos de l'ordonnance du TA de Cergy, 18 février $2020, n^{\circ} 2001274$

\section{Camille Bourdaire-Mignot and Tatiana Gründler}

\section{OpenEdition}

\section{Journals}

Electronic version

URL: http://journals.openedition.org/revdh/8967

DOI: 10.4000/revdh.8967

ISSN: 2264-119X

\section{Publisher}

Centre de recherches et d'études sur les droits fondamentaux

\section{Electronic reference}

Camille Bourdaire-Mignot and Tatiana Gründler, « Arrêt des traitements médicaux pour un patient âgé », La Revue des droits de l'homme [Online], Actualités Droits-Libertés, Online since 14 March 2020, connection on 07 November 2020. URL : http://journals.openedition.org/revdh/8967 ; DOI : https:// doi.org/10.4000/revdh.8967

This text was automatically generated on 7 November 2020.

Tous droits réservés 


\section{Arrêt des traitements médicaux pour un patient âgé}

Quelques réflexions à propos de l'ordonnance du TA de Cergy, 18 février 2020, n² 2001274

\section{Camille Bourdaire-Mignot and Tatiana Gründler}

1 Le 17 janvier dernier, un homme âgé de 74 ans fut admis en réanimation au centre hospitalier de Pontoise à la suite d'un accident vasculaire cérébral. Plongé dans un coma profond, le patient fut intubé et placé sous aide respiratoire simple ${ }^{1}$. Considérant que la situation pouvait relever de l'obstination déraisonnable interdite par la loi, le médecin en charge de ce patient décida d'engager, le 23 janvier, la procédure collégiale prévue par le Code de la santé publique ${ }^{2}$. Après plusieurs entretiens ( 23 et 29 janvier), au cours desquels une information sur la situation médicale du patient fut délivrée aux membres de sa famille, la réunion pluridisciplinaire réglementaire avec l'équipe de soins palliatifs et un médecin extérieur - le chef de service des soins palliatifs - eut lieu le 30 janvier. À son issue, il fut décidé à la fois une limitation des traitements pour l'avenir (absence de réanimation en cas d'arrêt cardio-respiratoire) et un arrêt des thérapeutiques actuellement fournies au patient (ventilation mécanique, alimentation et hydratation artificielles). Cette décision fut notifiée à la famille, conformément aux exigences du Conseil constitutionnel ${ }^{3}$. Le fils du patient saisit alors en référé le tribunal administratif de Cergy afin qu'il enjoigne au centre hospitalier de suspendre cette décision, ce qu'il fit par une ordonnance rendue le 18 février $2020^{4}$, après avoir sollicité l'avis d'un expert ${ }^{5}$.

2 Malgré une trame classique, qui inscrit cette décision dans le sillage de celles rendues antérieurement par le Conseil d'État relativement à l'arrêt de traitement au titre de l'obstination déraisonnable (I), l'ordonnance recèle quelques ambiguïtés qui méritent d'être levées ou, à tout le moins, soulignées (II). 


\section{I/ - Le classicisme du cadre d'analyse}

3 L'analyse du juge administratif est conforme à la jurisprudence antérieure du Conseil d'État, tant sur le plan procédural (1) que substantiel (2).

4 1. Procédure ad hoc désormais classique. Après avoir jugé que la condition d'urgence était remplie, le tribunal saisi dans le cadre du référé-liberté de l'article L 521-2 du Code de la justice administrative a commencé par ordonner, le 4 février, une expertise afin, d'une part, de décrire l'état clinique actuel du patient et son évolution depuis l'hospitalisation et, d'autre part, de se prononcer sur le caractère irréversible des lésions neurologiques, sur le pronostic clinique ainsi que sur la nécessité du maintien de l'assistance respiratoire mécanique. Éclairé par les conclusions de l'expert, le juge administratif a ensuite pu statuer en formation collégiale ${ }^{6}$ sur la demande. Compte tenu de son office particulier s'agissant d'une décision d'arrêt des traitements - portant atteinte de manière irréversible à la vie -, le juge des référés n'exige pas, pour ordonner sa suspension, que la décision médicale litigieuse porte une atteinte grave et manifestement illégale à une liberté fondamentale, mais se contente du fait qu'elle n'entre pas dans les hypothèses prévues par la loi

2. Reprise d'une jurisprudence établie. Sur le fond, cette ordonnance cherche clairement à s'inscrire dans le cadre fixé dès 2014 par le Conseil d'État à propos du cas de Vincent Lambert ${ }^{8}$ et confirmé, après les modifications législatives intervenues en $2016^{9}$, dans l'affaire dite Marwa ${ }^{10}$. Ainsi est-il rappelé que, pour prendre une telle décision, le médecin «doit se fonder sur un ensemble d'éléments, médicaux et non médicaux, dont le poids respectif ne peut être prédéterminé et dépend des circonstances particulières à chaque patient, le conduisant à appréhender chaque situation dans sa singularité $»^{11}$. Dans cette perspective, il est souligné dans le présent cas que les éléments médicaux doivent " couvrir une période suffisamment longue ", être analysés "collégialement » et porter tout à la fois sur "l'état actuel du patient ", sur son « évolution» depuis l'accident, sur la «souffrance et sur le pronostic ». Au titre des éléments non médicaux, la volonté que le patient aurait pu exprimer doit par ailleurs faire l'objet d'une " attention particulière ${ }^{12}$.

6 Si la structure du raisonnement est claire et conforme à la jurisprudence antérieure, la solution retenue - suspension de la décision initiale d'arrêt des traitements et proposition d'une décision alternative - n'est cependant pas dépourvue de toute ambiguïté.

\section{II/ - Une analyse non dénuée d'ambiguïté}

7 La lecture de la décision soulève en creux deux interrogations: l'une relative à l'incidence de l'âge du patient dans l'appréciation du délai dans lequel est intervenue la décision d'arrêt des traitements (1) et l'autre portant sur le véritable auteur de la décision alternative suggérée in fine (2).

8 1. Âge du patient : une fausse indifférence? En l'espèce, le patient devenu hors d'état de s'exprimer n'avait pas fait part de ses volontés ni dans les directives anticipées - contraignantes depuis 2016 -, ni auprès d'une personne de confiance - dont le témoignage aurait pu être recueilli ${ }^{13}$-, ni auprès de sa famille ou de ses proches ${ }^{14}$. Quant aux éléments d'ordre médical, ils ont certes fait l'objet d'une vigilance appuyée 
de la juridiction qui a désigné un expert, mais on peut s'étonner que rien ne soit dit sur le délai - relativement bref en l'espèce - de cette appréciation. L'exigence jurisprudentielle d'une période suffisamment longue pour l'examen des éléments médicaux peut-elle être considérée comme respectée quand la décision de limitation et d'arrêt de traitements est prise moins de quinze jours après la survenue de l'accident du patient et son hospitalisation? Le silence du juge sur ce point interroge.

Au premier abord la décision semble rassurante puisque le juge administratif y affirme clairement que "la seule circonstance qu'un patient, âgé, soit dans un état de coma "profond" sans espoir d'amélioration ne saurait caractériser, par elle-même, une situation dans laquelle la poursuite du traitement apparaîtrait injustifiée au nom du refus de l'obstination déraisonnable $»^{15}$. Pour autant, il n'est pas certain que le juge n'ait pas tenu compte de l'âge - relativement avancé - du patient lors de son appréciation de la situation, et singulièrement de son appréciation du délai dans lequel intervient la décision d'arrêt des traitements.

On se souvient en effet que dans l'affaire Marwa, qui concernait un bébé âgé de 12 mois au moment des faits, la décision d'arrêt des traitements - prise un mois et demi après l'hospitalisation de l'enfant en réanimation - n'a pas a été considérée comme adoptée " au terme d'un délai suffisamment long pour évaluer de manière certaine les conséquences de ses lésions neurologiques $\aleph^{16}$. Au contraire, pour un patient âgé de 79 ans, le Tribunal administratif de Lyon $^{17}$ a pu estimer suffisant le délai de trois semaines. Dans le présent cas, le juge, qui rappelle pourtant que « les éléments médicaux doivent couvrir une période suffisamment longue ${ }^{18}$, ne s'attarde pas sur le fait qu'en l'espèce, c'est seulement dix jours après son AVC que le patient a fait l'objet d'une décision médicale d'arrêt. Sans doute un pronostic fiable peut-il est établi plus rapidement pour un patient, dont l'âge avancé limite les capacités de récupération, que pour un tout jeune patient ${ }^{19}$. Mais ce qui est frappant, c'est l'absence de toute appréciation du délai en l'espèce. Si le juge fait preuve de sagesse en s'abstenant de fixer un délai minimum, il ne doit pas omettre de rechercher au cas par cas si les éléments médicaux recueillis couvrent une période suffisante pour établir un pronostic fiable. Il ne faudrait pas que, au prétexte de l'âge avancé du patient, les situations soient évaluées trop rapidement. Les futurs contentieux devront être examinés à l'aune du risque d'âgisme de la part des médecins comme des juges.

11 2. Un arrêt partiel des traitements... En l'espèce, le Tribunal de Cergy déduit des éléments relatifs à l'état clinique du patient, tels qu'éclairés par l'expert, que les conditions légales d'une décision d'arrêt des traitements au titre de l'obstination déraisonnable ne sont pas réunies et enjoint donc au centre hospitalier la suspension de ladite décision tout en lui suggérant - de manière assez inhabituelle - une décision alternative d'arrêt partiel des traitements. L'ordonnance ne précisant pas les éléments problématiques de la décision dont elle ordonne la suspension, on peut formuler l'hypothèse que le juge a en réalité pris en considération le souhait de la famille relatif aux conditions de la fin de vie du patient et proposé, pour cette raison, à l'hôpital une autre décision "consistant en une extubation et un maintien de l'hydratation et de l'alimentation, quelle que soit la réaction [du patient] $\aleph^{20}$. Il est vrai que dans son mémoire fourni à la suite de l'expertise la préconisant, le centre hospitalier avait affirmé son accord pour cette solution. Il est vrai aussi que dans ces situations difficiles, le juge se montre particulièrement attentif à la mise en œuvre des décisions qu'il rend dans un souci d'apaisement des relations soignants/famille. Ainsi, par le passé, il a pu, 
alors même qu'il validait une décision médicale d'arrêt des traitements, préciser qu'il « appartenait au médecin d'apprécier, compte tenu de l'ensemble des circonstances de l'espèce, si et dans quel délai la décision d'arrêt de traitement $\mathrm{d}$ [evait] être exécutée ${ }^{21}$. Ici, la perspective est sensiblement différente puisque les magistrats viennent nuancer la portée de leur remise en cause de la décision médicale en offrant des pistes alternatives à l'équipe médicale. Cela semble néanmoins participer du même souci de bienfaisance à l'égard non seulement du patient, ce qui est une obligation légale et éthique, mais aussi à l'égard des familles. Et c'est là que l'ordonnance interroge. Qui pourra in fine être considéré comme ayant pris la décision dont dépend la fin de vie du patient? Le médecin, comme les textes l'imposent, la famille dont le souhait que son proche meure « dans des conditions naturelles » a été entendu, l'expert qui a proposé le premier cette alternative ou le juge qui la suggère alors qu'il aurait pu se contenter de suspendre la décision d'arrêt?

... pour une " mort naturelle ". En l'espèce, famille et soignants ne sont pas en totale opposition puisqu'ils semblent tous admettre que l'issue pour le patient est la mort. Ils divergent en revanche sur les conditions précédant cette issue. La famille souhaite que son proche "décède d'une mort naturelle» et paraît considérer que l'arrêt des traitements précipiterait (artificiellement) la mort ${ }^{22}$. Au contraire, pour le médecin, c'est précisément le maintien des soins de réanimation qui empêche cette mort naturelle. Pourtant, dans ses observations orales, celui-ci se range à la position de compromis proposé par l'expert. De même, le centre hospitalier se montre favorable à l'arrêt de la ventilation tout en maintenant le traitement antalgique ainsi que l'alimentation et l'hydratation artificielles «de façon à placer les conditions physiologiques normales et naturelles, répondant ainsi au souhait de la famille (...) d'une mort «naturelle» ${ }^{23}$. À cette prise en compte des souhaits des proches par l'expert, puis, par le médecin et, enfin, par le juge, semble devoir s'ajouter la prise en compte du temps qui lui est nécessaire pour accepter l'issue fatale. Ce qui nous renvoie à la question du délai entre le début de la prise en charge médicale du patient et la décision d'arrêt des traitements au titre de l'obstination déraisonnable. Encore une fois, si les médecins sont en mesure, pour une personne d'un âge relativement avancé, d'apprécier - dans un temps court - le caractère irréversible ou non de l'état du patient ${ }^{24}$, le temps d'acceptation de la famille n'est pas pour autant accélérée ${ }^{25}$. Dans le cas du nourrisson, de l'enfant ou du jeune adulte en fin de vie, l'opposition éventuelle des parents à la décision d'arrêt des traitements traduit le plus souvent l'impossibilité d'accepter la mort de leur enfant. Pour un parent âgé, comme c'est le cas ici, l'opposition est d'une autre nature car le décès semble davantage dans l'ordre des choses. Mais la manière dont le patient s'éteint reste importante. C'est sans doute ce qu'exprimaient les proches à travers le vœu d'une fin naturelle pour leur proche. Il apparaît important que le médecin tienne compte d'un tel élément dans le temps dédié à la prise de décision de limitation ou d'arrêt des traitements ainsi que dans les modalités proposées pour une telle limitation ou un tel arrêt. C'est en effet à cette condition que la décision prise dans l'intérêt du patient sera acceptée par ses proches. En revanche, le fait que le juge semble également tenir compte du ressenti des proches pour apprécier les conditions légales de la prise de décision médicale interroge davantage. Il ne faudrait pas que la bienveillance à l'égard de la famille prenne le pas sur l'intérêt supérieur du patient en fin de vie. 
Les Lettres « Actualités Droits-Libertés » (ADL) du CREDOF (pour s'y abonner) sont accessibles sur le site de la Revue des Droits de l'Homme (RevDH) - Contact

\section{NOTES}

1. Cela signifie que l'aide respiratoire ne se déclenche qu'en fonction des besoins du patient.

2. Article L 1110-5-1 alinéa 1 et article R 4127-37-2 III du Code de la santé publique.

3. Exigence posée par le Conseil constitutionnel pour garantir l'effectivité du droit au recours : « le droit à un recours juridictionnel effectif impose que cette décision soit notifiée aux personnes auprès desquelles le médecin s'est enquis de la volonté du patient, dans des conditions leur permettant d'exercer un recours en temps utile " (Conseil constitutionnel, n² 2017-632 QPC du 2 juin 2017).

4. TA Cergy-Pontoise, 18 février 2002, $n^{\circ} 2001274$.

5. TA Cergy-Pontoise, 4 février 2020, $n^{\circ} 2001274$.

6. Conformément à ce que permet l'article L 511-2 du Code de la justice administrative.

7. Point 16 : «les conditions prévues par la loi pour que puisse être prise, par le médecin en charge du patient, une décision mettant fin à un traitement n'ayant d'autre effet que le maintien artificiel de la vie et dont la poursuite traduirait ainsi une obstination déraisonnable ne peuvent être regardées, dans le cas de M. T., comme réunies ».

8. CE, Ass., 24 juin 2014, Mme Lambert, $n^{\circ} 375081,375090$ et 37509.

9. Loi $n^{\circ} 2016-87$ du 2 février 2016 créant de nouveaux droits en faveur des malades et des personnes en fin de vie.

10. CE, 8 mars 2017, Assistance publique - Hôpitaux de Marseille, $\mathrm{n}^{\circ} 408146$.

11. Point 10.

12. Point 10.

13. Point 14.

14. Voir sur ce point les observations orales du requérant, M. S., fils du patient.

15. Point 16. Nous soulignons.

16. Point 22.

17. TA Lyon, ord. 9 novembre $2016, n^{\circ} 1607855$, point 12 . Le patient avait été accueilli à l'hôpital de la Croix Rousse de Lyon le 7 octobre 2016 et la décision d'arrêt des traitements fut prise le 28 octobre, soit 21 jours après.

18. Point 10.

19. Certains médecins ont pu souligner le risque de biais chez les très jeunes patients dans l'appréciation de l'évolution de leur état depuis l'accident ou la maladie car, malgré l'importance de ses lésions neurologiques, des progrès seront presque toujours observés en raison de leur phase de forte croissance.

20. Point 16. 
21. CE 5 janvier 2018, Mme B et M. D, n 416689.

22. Le requérant souhaite que son père reste sous aide respiratoire jusqu'à ce qu'il décède « de manière naturelle ».

23. Point 16. Nous soulignons.

24. Il résulte du dossier quotidien du patient que « la question du caractère suffisant du délai ou non a été évoquée par la fille du patient » et «qu'il y a été répondu » (point 11). Ainsi, si le juge n'a pas expressément réinterrogé le délai, la question avait été soulevée et discutée avec la famille.

25. C'est ce qu'a compris l'équipe médicale qui, après avoir soutenu que la procédure collégiale de décision d'arrêt de soins avait été réalisée conformément à la loi de 2016, a indiqué «que la famille avait besoin de temps » et être « en conséquence prêt[e] à réexaminer la situation sur la base de la proposition de l'expert (...) », laquelle porte finalement non pas tant sur l'arrêt des traitements que sur les modalités de l'arrêt.

\section{ABSTRACTS}

De façon heureusement moins médiatique que pour l'affaire Lambert, des décisions médicales d'arrêt de traitement au titre de l'obstination déraisonnable continuent d'être soumises par les familles au contrôle du juge. Ainsi, le 18 février 2020, le TA de Cergy a eu à se prononcer en référé sur une telle décision prise à l'égard d'un patient âgé de 74 ans, pour lequel il a éprouvé le besoin de préciser que " la seule circonstance qu'un patient, âgé, soit dans un état de coma "profond » sans espoir d'amélioration ne saurait caractériser, par elle-même, une situation dans laquelle la poursuite du traitement apparaîtrait injustifiée au nom du refus de l'obstination déraisonnable ». Si l'affirmation se veut rassurante, la motivation de l'arrêt soulève toutefois quelques interrogations sur l'impact de l'âge avancé du patient sur la décision d'arrêt des traitements ainsi que sur les véritables auteurs d'une telle décision.

\section{AUTHORS}

\section{CAMILLE BOURDAIRE-MIGNOT}

Maîtresse de conférences en droit privé à l'Université Paris Nanterre (CEDCACE)

\section{TATIANA GRÜNDLER}

Maîtresse de conférences en droit public à l'Université Paris Nanterre (CTAD-CREDOF) 